\title{
Application of Organometallic Chemistry to the Electrical Interconnection of Graphene Nanoplatelets
}

Mingguang Chen, Xiaojuan Tian, Wangxiang Li, Elena Bekyarova, Guanghui Li, Matthew Moser, Robert C. Haddon*

Table S1. Abbreviations used in the paper

\begin{tabular}{lll}
\hline Number of Spectra & Abbreviation & Full name \\
\hline (1) & EG-SG & Epitaxial Graphene-Single Layer Graphene \\
(2) & CVD-SG & Chemical Vapor Deposition-Single Layer Graphene \\
(3) & XSG Flake & Mechanically Exfoliated Single Layer Graphene Flake \\
(4) & XBG Flake & Mechanically Exfoliated Bilayer Graphene Flake \\
(5) & XFG Flake & Mechanically Exfoliated Few layer Graphene Flake \\
(6) & PPG GNP & PPG Graphene Nanoplatelet \\
(7) & XG Powder & Solvent Exfoliated Graphene Powder \\
(8) & GNP & Common Graphene Nanoplatelet \\
(9) & ODA-G & Octadecylamine Functionalized Graphene \\
(10) & RGO & Reduced Graphene Oxide \\
(11) & GO & Graphene Oxide \\
\hline
\end{tabular}

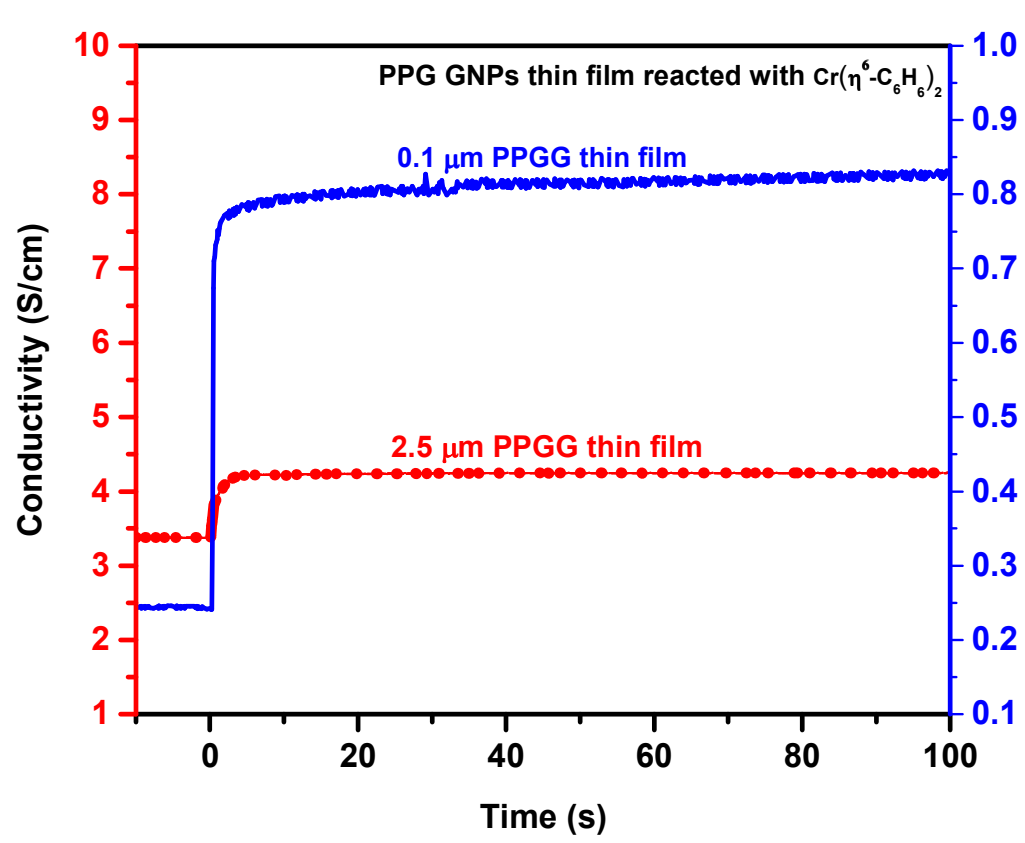

Figure S1 Effect of film thickness on the photochemically induced conductivity enhancement of the PPG GNP thin films.

In Figure S1, the initial conductivity of the thick film $\left(\mathrm{t}=2.5 \mu \mathrm{m}, \mathrm{d} \approx 0.77 \mathrm{~g} / \mathrm{cm}^{3}\right)$ is higher than that of the thin film $\left(t=0.1 \mu \mathrm{m}, \mathrm{d} \approx 0.29 \mathrm{~g} / \mathrm{cm}^{3}\right)$ because of the more efficient percolation of the carriers. ${ }^{1}$ The 
response of the thick film to the organometallic reagent is less than that of the thin film for the same reason, and because of the difficulties experienced by the reagent and the light in penetrating

throughout the bulk film.

(1) Tian, X.; Moser, M. L.; Pekker, A.; Sarkar, S.; Ramirez, J.; Bekyarova, E.; Itkis, M. E.; Haddon, R. C., Effect of Atomic Interconnects on Percolation in Single-Walled Carbon Nanotube Thin Film Networks. Nano Lett. 2014, 14, 3930-3937. 